\title{
Biomechanical changes at the ankle joint after stroke
}

\author{
A F Thilmann, S J Fellows, H F Ross
}

\begin{abstract}
The resistance of the relaxed ankle to slow displacement over the joint movement range was measured on both sides of a group of hemiparetic stroke patients, in whom spasticity had been established for at least one year and who showed no clinical signs of contractures. The ankle joints of the age-matched normal subjects were flexible over most of the movement range, showing dramatically increasing stifiness only when the foot was dorsiflexed beyond $70^{\circ}$, with a neutral range between $90-110^{\circ}$, and $a$ less dramatic increase in stiffness during plantarflexion. Hemiparetic patients showed identical curves to the normal subjects on the "healthy" side, ipsilateral to the causative cerebral lesion, but were significantly stiffer in dorsiffexion on the contralateral side, without change in the minimum stiffness range or during plantarflexion. Therefore significant changes in passive biomechanical properties occur at the affected ankle of hemiparetic subjects, predominantly as the result of a loss of compliance in the Achilles tendon, although an increase in the passive stifiness of the triceps surae may also occur. The contribution of these changes to the locomotor disability of hemiparetic patients is discussed.
\end{abstract}

One of the major disabilities associated with spasticity in humans is muscle hypertonia which, to a varying degree, can hinder or even prevent voluntary movements. Classically, hypertonia is caused by a velocity dependent increase in stretch reflex mechanisms. ${ }^{1}$ In recent years, however, evidence has shown that spastic hypertonia cannot invariably be explained by stretch reflex activity. It has been observed ${ }^{2}$ that during the swing phase of gait, the tibialis anterior (TA) muscle shows abnormally high levels of activity, despite the lack of any EMG activity in its antagonist, the triceps surae (TS) muscle. This was interpreted as evidence of pathological changes in mechanical muscle fibre properties in the TS which led to increased resistance to dorsiflexing movements. An increase in the proportion of type I fibres in the TS, due to type II fibre atrophy has been observed in paretic muscle, ${ }^{3}$ but while this is likely to affect the active mechanical properties of the muscle, no difference in the passive properties of the TS would be expected and thus the origin of the reduced flexibility of the ankle joint remains unclear.

It has also been reported ${ }^{4}$ that subjects in whom spasticity had been present for a considerable time show enhanced stiffness at the ankle in the absence of reflex activity. This result was complicated by the presence of mild to moderate contractures in the majority of the subjects involved in the study, and the wide range of causes from which the spasticity arose. Recently it has been shown that in hemiparetic subjects with no clinical evidence of contracture, enhanced muscle tone in the elbow musculature is always associated with pathological stretch reflex activity. ${ }^{5}$ Given the wide differences in reflex function between the upper and lower limbs it is possible that the mechanisms of muscle hypertonia might also differ between the two. Accordingly, this study was carried out to establish if alterations in passive biomechanical properties do occur at the ankle in subjects with no clinical evidence of contracture and with spasticity arising from a well defined cause (unilateral cerebral ischaemic lesion).

The findings show that significantly greater stiffness is seen during dorsiflexion in the ankle contralateral to the lesion in hemiparetic subjects. The functional significance of these changes, however, is difficult to interpret: in a normal population wearing shoes, it is estimated ${ }^{67}$ that between $30-70 \%$ show some anatomical abnormalities of the foot which, while leading to no functional problems and requiring no treatment beyond the use of insoles, ${ }^{8}$ are often associated with reduced flexibility at the ankle. Such subjects are easily identified clinically, and when the passive resistance of the ankle was measured in such a group it was found that they show significantly greater passive resistance at the ankle to imposed dorsiflexion than does the affected side of the hemiparetic subjects. Part of these results has been reported briefly elsewhere. ${ }^{9}$

\section{Methods}

Subjects

This study was performed on 15 hemiparetic human subjects, mean age 47.7 years, (SE) 3.9 , who had suffered a unilateral ischaemic cerebral lesion in the area of the right or left middle cerebral artery at least one year before the experiments took place. None of the patients was receiving anti-spasticity medication, but all were having regular physio- 
therapy at least once a week. Full clinical details are given in the table. In addition, two groups of normal subjects were studied. One group, of ten subjects, mean age $44 \cdot 2$ years, (SE) $3 \cdot 6$, showed no history of neurological or orthopaedic problems. The other group of five subjects, while being in all other respects normal, were orthopaedically assessed as having abnormal feet, without associated functional problems. Such abnormalities which may arise from a variety of anatomical causes (such as pes equinus, pes planus, pes planovalgus etc), have been estimated to occur in up to $70 \%$ of the population. ${ }^{7}$ These subjects will be referred to as the "stiff" normal group. The "stiff" normal group had normal gait patterns, experienced no pain, engaged in sport and were not generally overweight. Aside from orthopaedic considerations, the only clear visible difference between them and the flexible normal group was that the "stiff" normal subjects were all unable to squat on their heels, although all the flexible normal subjects could do so without trouble.

All subjects gave their informed consent to the procedures, which had been approved by the local ethical committee.

\section{Procedures}

The experimental set-up is shown in fig 1 .

The subjects were seated in a heavy, stable chair and the leg to be tested was placed in the equipment. With the knee flexed $\left(105^{\circ}\right)$, the foot was fixed in a cast, where the position could be altered relative to a bearing mounted stirrup, so that the anatomical axis of the ankle was aligned with the axis of rotation of the bearings. This was assessed by the lack of palpable movement in the tibia during slow, small amplitude, sinusoidal displacement of the ankle. The knee joint was then also fixed in place. A rigid connecting rod linked the stirrup to another set of bearings, which could be moved by means of a lever. Manual rotation could then be made over the entire movement range of the ankle. These movements were not motorised as it was considered that the experimenter could assess more easily the limits of movement for a given subject and thus avoid discomfort or injury that might arise from the use of a stereotyped, computer generated movement. The torque generated in resistance to the applied displacement was monitored by a piezo-element (Kistler, 9311a) mounted in series with the

Figure 1 Experimental set-up. See text for explanation.

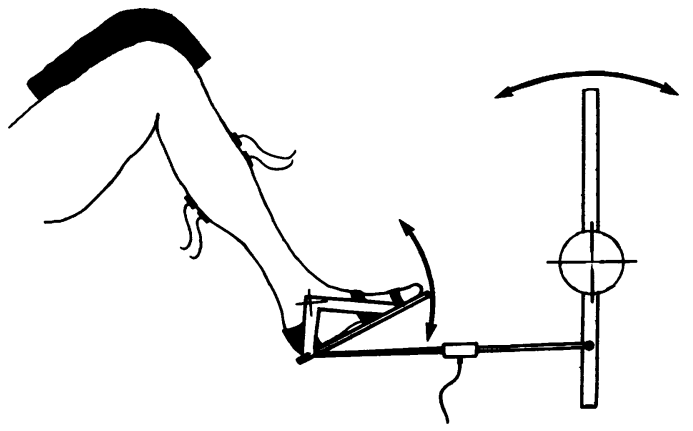

connecting rod. Position was indicated by a potentiometer (Megatron, MCP40) mounted at the axis of rotation of the ankle. Surface EMG recordings were made from the TS and TA muscles and after amplification and filtering (bandwidth $10 \mathrm{~Hz}-1 \mathrm{kHz}$ ) through a custom-built EMG amplifier, were passed, along with torque and position records, to the FM channels of a tape recorder (Racal Store7D). The EMG was also rectified and passed, with force and position records, to the ADC boards of a laboratory computer (DEC, PDP 11/73), which sampled each channel at $100 \mathrm{~Hz}$ and stored the data on disc for later analysis.

At the start of an experimental sequence the ankle was placed in the so-called neutral position. This was defined as a $90^{\circ}$ angle between the anterior margin of the tibia and the sole of the foot. Force and position records were zeroed at this point. The experimenter then applied a series of five slow linear ramp movements with a stretch velocity of 5-8 $\operatorname{deg~~^{-1}}$, each movement going first into dorsiflexion, then back to the limit of plantarflexion, returning finally to the neutral position (around 75 seconds per movement cycle). During the entire procedure the subjects were asked to remain relaxed and not to interfere with the applied movements. We confirmed that these instructions were complied with by monitoring the EMG records. A typical sequence of movements is shown in fig 2 . It can be seen that the experimenter was able to apply regular and reproducible ramp stretches. One movement cycle is defined by the two cursors on the position record. The velocity curve was constructed from the position record by the computer.

\section{Analysis}

After the completion of an experimental sequence, and having eliminated any movement cycles containing EMG activity, the position and torque records were processed for each movement cycle to yield the stiffness of the ankle over the range of movement. This was done by dividing the range of movement into $5^{\circ}$ bins. All the data points falling within a bin were then used to fit the force as a straight line function of position. The slope of this line thus provided the stiffness of the joint for the points in each bin. Flexing and extending movements were processed separately: the results quoted in this study came from the first full flexing movement between $130^{\circ}$ and $60^{\circ}$.

Differences in stiffness between the side ipsilateral and that contralateral to the cerebral lesion were then assessed for corresponding movements using a two-tailed, paired $t$ test. Differences between the hemiparetic subjects and the two groups of neurologically normal subjects were assessed using variance analysis.

\section{Results}

Analysis of the data in the fully normal group revealed a characteristic, rather flat-bottomed, U-shaped curve of stiffness over the movement range (fig 3 ). 

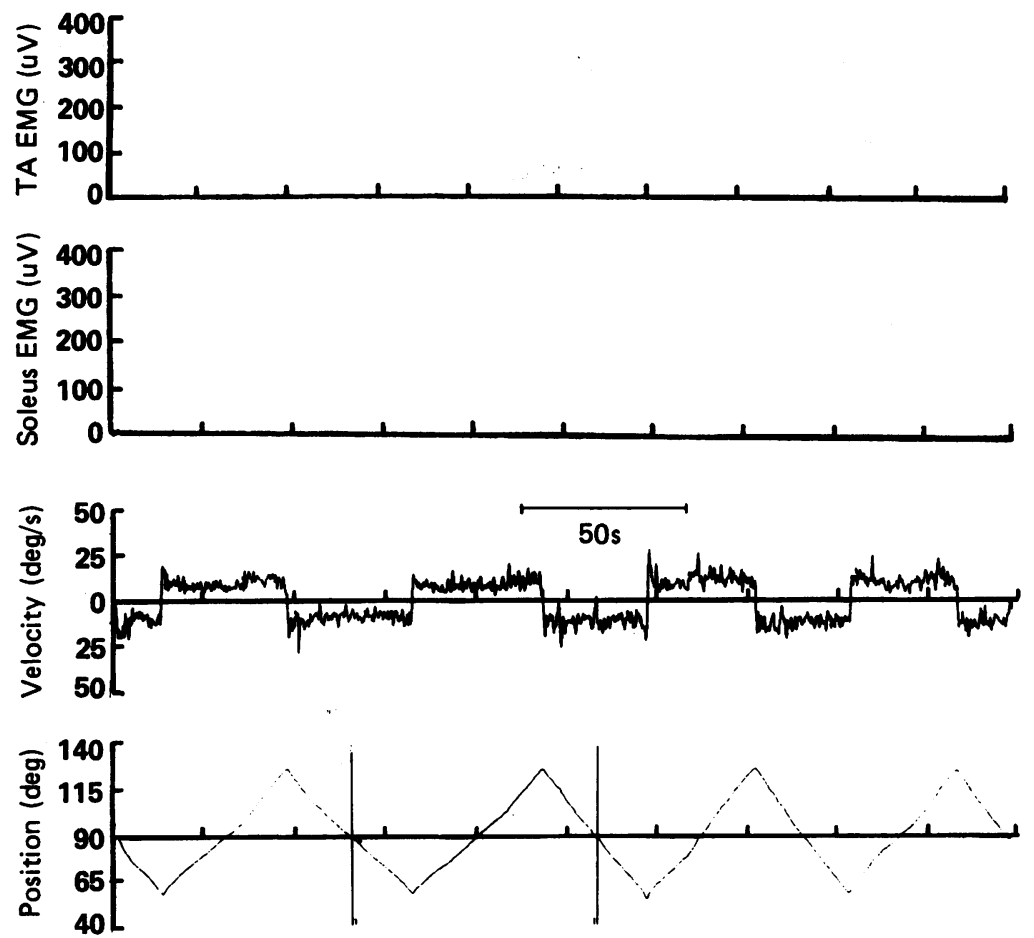

Figure 2 A typical movement sequence showing, from the bottom up, the position of the ankle joint, the velocity of the applied stretch, TS EMG and TA EMG. Note that the applied displacements were highly linear and very slow. Single movement cycles were subsequently selected for further analysis (a single cycle is marked by the vertical cursors on the position trace).

It is apparent that this group shows little passive resistance over a wide part of the movement range, with a minimum resistance between 90 and $110^{\circ}$. As the foot is moved into dorsiflexion, the stiffness increases slowly until $70^{\circ}$, when it begins to increase more steeply. Moving the foot into dorsiflexion beyond $60^{\circ}$ was possible in these subjects, but was an uncomfortable experience and often led to activation of the TA (at a time when this muscle was at its shortest length). If the foot was plantarflexed, it may be seen that the stiffness first began to increase significantly beyond $110^{\circ}$. The limit of $130^{\circ}$ was determined by the mechanical construction of the equipment, and

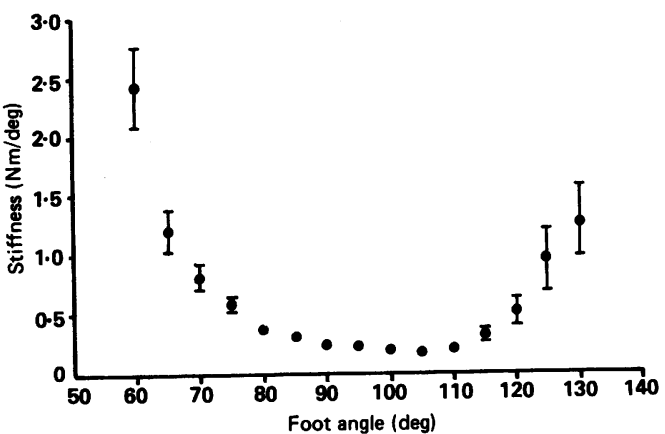

Figure 3 Mean (SE) stiffnesses over the movement range of the ankle obtained from the force and position records of the first movement cycle applied to the flexible normals $(n=10)$

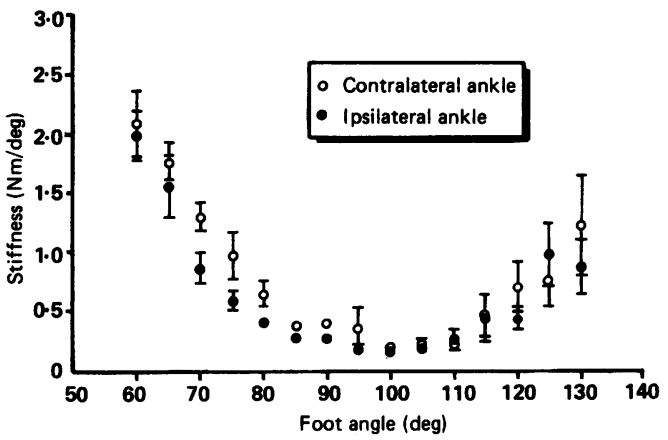

Figure 4 Mean (SE) stiffnesses over the movement range of the ankle for both sides of the hemiparetic subjects $(n=15)$. Ipsi and contralateral are in reference to the affected cerebral hemisphere.

not necessarily by anatomical limitations of the subject's ankle. This curve is compatible with most of those obtained in an earlier study ${ }^{10}$ by the application of small, pseudo-random perturbations around different mean angles over the movement range, although at least one of the subjects in this earlier study (number two, table) ${ }^{10}$ would be classified as a "stiff" normal (see below) on the basis of their restricted movement range and considerably higher resistance to dorsiflexion.

Table Clinical details of the hemiparetic subjects

\begin{tabular}{|c|c|c|c|c|c|c|c|c|c|}
\hline \multirow[b]{2}{*}{ Case } & \multirow[b]{2}{*}{ Sex } & \multirow{2}{*}{$\begin{array}{l}\text { Age } \\
\text { (years) }\end{array}$} & \multirow[b]{2}{*}{$S L^{1}$} & \multirow{2}{*}{$\begin{array}{l}\text { Duration } \\
\text { (months) }\end{array}$} & \multirow[b]{2}{*}{$P^{2}$} & \multirow[b]{2}{*}{$M^{3}$} & \multicolumn{2}{|l|}{$A T R^{4}$} & \multirow{2}{*}{$\begin{array}{l}\text { Muscle } \\
\text { tone }\end{array}$} \\
\hline & & & & & & & $c$ & $i$ & \\
\hline $\begin{array}{r}1 \\
2 \\
3 \\
4 \\
5 \\
6 \\
7 \\
8 \\
9 \\
10 \\
11 \\
12 \\
13 \\
14 \\
15\end{array}$ & $\begin{array}{l}m \\
f \\
f \\
f \\
f \\
f \\
m \\
m \\
m \\
m \\
m \\
m \\
m \\
f \\
m\end{array}$ & $\begin{array}{l}48 \\
50 \\
48 \\
24 \\
19 \\
50 \\
34 \\
45 \\
66 \\
68 \\
57 \\
60 \\
58 \\
52 \\
36\end{array}$ & $\begin{array}{l}\text { 1.MCA } \\
\text { 1.MCA } \\
\text { 1.MCA } \\
\text { r.MCA } \\
\text { r.MCA } \\
\text { r.MCA } \\
\text { r.MCA } \\
\text { r.MCA } \\
\text { r.MCA } \\
\text { 1.MCA } \\
\text { I.MCA } \\
\text { r.MCA } \\
\text { I.MCA } \\
\text { r.MCA } \\
\text { r.MCA }\end{array}$ & $\begin{array}{l}48 \\
72 \\
13 \\
16 \\
14 \\
13 \\
20 \\
25 \\
13 \\
15 \\
12 \\
12 \\
18 \\
18 \\
23\end{array}$ & $\begin{array}{l}1 \\
1 \\
1-2 \\
4 \\
4 \\
2-3 \\
4 \\
0 \\
5 \\
4 \\
0 \\
4-5 \\
0-1 \\
5 \\
4\end{array}$ & $\begin{array}{l}5 \\
5 \\
5 \\
5 \\
5 \\
2 \\
5 \\
2 \\
5 \\
2 \\
0 \\
5 \\
4 \\
5 \\
4-5\end{array}$ & $\begin{array}{l}+ \\
+ \\
++++ \\
+++ \\
+++ \\
+++ \\
++++ \\
++++ \\
- \\
++++ \\
++++ \\
++++ \\
++++ \\
++ \\
+++\end{array}$ & $\begin{array}{l}(+) \\
+ \\
++ \\
+ \\
+ \\
+ \\
+ \\
+ \\
+ \\
+ \\
++ \\
+ \\
+ \\
+ \\
+\end{array}$ & $\begin{array}{l}3 \\
3 \\
1 \\
0 \\
0 \\
1 \\
1-2 \\
1 \\
0 \\
0 \\
0 \\
1 \\
2 \\
0 \\
0\end{array}$ \\
\hline
\end{tabular}

'Site of lesion:I.MCA = left middle cerebral artery. r.MCA = right middle cerebral artery. Lesion localisation in all cases by CT plus, in some cases, MRI and/or angiography.

'Paresis of contralateral TS, expressed according to the British MRC scale: $0=$ no voluntary power; $1=$ visible contraction without effect; $2=$ movement without the influence of gravity; $3=$ movement against gravity; $4=$ movement against resistance; $5=$ normal power.

'Mobility: $0=$ no mobility; $1=$ with two crutches; $2=$ with one crutch; $3=$ unaided, up to $100 \mathrm{~m} ; 4=$ unaided, up to $1 \mathrm{Km} ; 5=$ unaided, more than $1 \mathrm{Km}$. tAchilles tendon reflex on the contralateral (c) and ipsilateral (i) side: $-=$ absent; $(+)=$ weak; $+=$ normal; $++=$ brisk normal; $+++=$ exaggerated with beats of clonus; $++++=$ exaggerated with sustained clonus.

"Muscle tone of the contralateral leg (assessed during passive knee flexion and extension), expressed on the Ashworth scale: $0=$ no increase in tone; $1=$ slight increase in tone giving a "catch" when the limb was moved in flexion or extension; $2=$ more marked increase in tone but limb easily flexed; $3=$ considerable increase in tone-passive movement difficult; $4=$ limb rigid in flexion or extension. 
Figure 5 Mean ( $S E$ ) stiffnesses over the movement range of the ankle for the "stiff" normal subjects $(n=5)$ and the contralateral side of the hemiparetic subjects $(n=15)$.

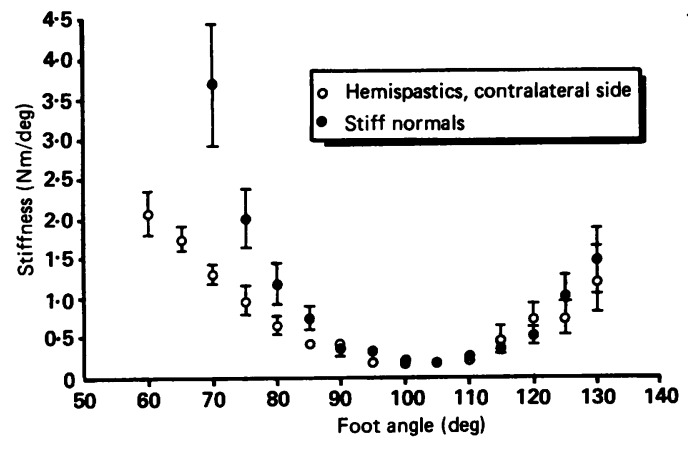

Figure 4 shows a similar curve calculated for the side ipsilateral to the cerebral lesion in the hemiparetic subjects (filled circles), superimposed on the curve for the contralateral ankle (open circles). In plantarflexion and between 95 and $110^{\circ}$, there were no differences between the two body sides. As the ankle is dorsiflexed, however, the contralateral ankle is significantly stiffer $(p<0.01)$ at all angles between 90 and $70^{\circ}$. The range of movement possible at the joint was unchanged from normal values. The increase in stiffness in dorsiflexion was evident in all the spastic subjects. The stiffness curve of the ipsilateral ankle of the hemiparetic subjects is largely identical to that of the flexible normal subjects.

While the stiffness of the contralateral ankle in the hemiparetic subjects is significantly greater than both their ipsilateral side and the flexible normal ankle, it can be seen (fig 5) that it is significantly less stiff ( $p<0.001)$ for all angles between 70 and $85^{\circ}$ than that of the ankle of the "stiff" normal subjects who form a homogeneous group with a characteristic stiffness profile. The movement range of the ankle of the "stiff" normal group is limited, and dorsiflexion beyond $70^{\circ}$ is impossible. The range of minimum stiffness and the stiffness in plantarflexion are the same as those observed in the flexible normal group and on both sides of the spastic subjects.

\section{Discussion}

For a hemiparetic patient it is a normal experience when walking that the toes drag on the ground during the swing phase of the paretic leg. From gait studies, ${ }^{2}$ this is known to result from an insufficient dorsiflexion of the ankle. The present findings, of significantly greater passive resistance to dorsiflexion at the paretic ankle could well represent the pathophysiological basis for this motor disability: at an angle of $80^{\circ}$ dorsiflexion, which must routinely be achieved in normal gait, ${ }^{11}$ the spastic ankle shows over $50 \%$ more resistance to imposed displacement than the normal ankle.

The classic view of spastic muscle hypertonia is that it arises as the result of exaggerated stretch reflex activity. While recent reports ${ }^{512}$ have confirmed that, at the elbow, spastic muscle hypertonia is always associated with velocity-dependent tonic EMG activity, this is clearly not the case at the ankle, where a significant contribution to raised tone arises from changes in the passive properties of the ankle. A contribution of stretch reflex activity to raised muscle tone at the spastic ankle cannot, however, be excluded as a result of this study: displacement was deliberately applied at low velocities of stretch to prevent the generation of reflex EMG in the ankle musculature. Whether velocity-dependent tonic EMG activity such as that seen at the elbow can also be seen at the ankle with faster rates of displacement is the subject of continuing research: preliminary results indicate abnormal responses are more likely to take the form of bursts of clonus.

All of the patients involved in this study were screened to exclude clinically defined contracture from the results and all cases of activation of the ankle musculature were strictly excluded. It is clear therefore that the observed increases in stiffness must arise as the result of passive biomechanical changes. Given that no differences from the normal condition were found in plantarflexion, this increased stiffness must be a property of the ankle extensor musculature or of its tendons and connective tissue. Changes in the bones of the ankle joint can be excluded as a major cause, as significant increases in stiffness were apparent at $85^{\circ}$, well away from any anatomical restraints likely to be imposed by the bones of the joint. The ligaments of the joint may similarly be excluded, as they are designed to act only at the extreme of the movement range, not otherwise being under tension ${ }^{13}$ and are more likely to represent a distinct boundary rather than the steady increase in stiffness observed, with an associated reduction in the movement range, which was not observed in the spastic subjects.

Thus it would seem likely that the changes underlying the increased stiffness lie, as previously suggested, ${ }^{24}$ in altered mechanical properties of the TS muscle group and/or its tendon. Both earlier reports favoured changes in the muscle fibres as the probable cause, but given the differing Young's moduli of muscle and tendon, the far greater proportional length (6-8 times) and the smaller cross-sectional area (about 140 times) $^{14}$ of the latter, it is more likely that changes in the Achilles tendon (that is, all connective tissue in line with a muscle fibre between its origin and insertion) have led to the observed increase in stiffness. A dorsiflexion of $10^{\circ}$ at the ankle would displace the insertion of the Achilles tendon (the calcaneus) approximately $8 \mathrm{~mm} .{ }^{15}$ The tendon would normally be expected to be highly elastic, rather than viscous, ${ }^{16}$ show high compliance ${ }^{17}$ and be proportionally much longer than its muscle fibres. Such a displacement would be well within its lengthening range: even if the muscle fibres themselves were rigid, a minimal increase in resistance would be seen, as the movement would simply be taken up in the tendon. That the dramatic increase in stiffness (over $50 \%$ ) was observed at $80^{\circ}$ of dorsiflexion means therefore that alterations in the stiffness of the Achilles tendon must have taken place in the spastic subjects.

From animal experiments it is known that immobilisation for periods as short as nine 
weeks can lead to significant stiffening of the joint, ${ }^{18}$ probably as the result of water loss in tendon and connective tissue, as well as a small amount of extra collagen deposition. This is unlikely to explain our results as all the subjects were having regular physiotherapy, and had been since shortly after their stroke. Furthermore, no correlation could be found between either the degree of paresis or the mobility of a subject and the stiffness, making it unlikely that simple disuse could be the cause of the changes. Given that the half-life of collagen is $300-500$ days, ${ }^{19}$ it is possible that some alteration in the collagen content of the Achilles tendon or other connective tissue has occurred: deposition of even a small amount of collagen outside the tissue weave has a marked effect on the physical characteristics of connective tissue. ${ }^{18}$ It is also thought that the so-called tendon slack, which accounts for the toe region of the tendon length/tension profile, is a property of the mucopolysaccharides contained in the tendon. ${ }^{20} \mathrm{~A}$ loss of flexibility in these structures might also be expected to lead to increased stiffness. There is still no evidence for such morphological changes in spastic humans.

Cooling a limb with ice packs or in a cold water bath $\left(10^{\circ} \mathrm{C}\right)$ leads to a marked increase in the passive resistance to imposed movements. ${ }^{21}$ Given that many of the patients in this study had moderate to severe paresis in the muscles of the lower leg, it is possible that a drop in intramuscular temperature might have occurred in these muscles, due to their relative disuse, and that this temperature drop has led to increased stiffness in the muscles. While the skin temperature of the patients in this study was not systematically measured, the clinician noted no differences between the two sides of the hemiparetic patients during the neurological examination that preceded each experiment. Furthermore, it has been shown that the skin temperature over the calf muscles in fully plegic patients is around $32^{\circ} \mathrm{C} .{ }^{22}$ As the temperature of the underlying muscles must be still higher, it is unlikely that the effects of cold on muscle stiffness can explain the observed increase in stiffness.

The patients selected for this study were healthy, mostly mobile and all were having regular physiotherapy using a wide variety of techniques. Nevertheless, a clear increase in the stiffness of the ankle contralateral to the site of the causative lesion was apparent in all of them and to a similar extent. Clinical experience indicates that subjects receiving no physiotherapy and in a generally unhealthy condition experience worse disability, developing considerably increased stiffness and a restricted movement range within a few months of their stroke. In these cases, pathological changes resulting from the mechanisms mentioned above may well play a more important role. It is clear from the failure to lift the forefoot in walking that this increased stiffness disables even our relatively healthy group of paretic subjects to some extent. What is less clear is why they are unable to correct for this stiffness. Despite showing significantly greater resis- tance than the paretic subjects and, additionally, a restricted movement range in dorsiflexion, the "stiff" normal group have a normal gait pattern: it is clear that such an increase in stiffness can be overcome. That the paretic subjects fail to do so may be a property of the automatic spinal circuits controlling locomotion.

The "stiff" normal group learnt, as children, to deal with the stiffness of their ankles as part of the maturation of gait. The paretic subjects, however, first encounter an increase in stiffness as adults, when the locomotor pattern is already established. It may be that the activity associated with this pattern is relatively inflexible and although some increase in the level of TA EMG output is possible, ${ }^{2}$ as reflected in recordings, it remains insufficient to overcome the increased passive stiffness of the Achilles tendon.

It must also be considered that a major component of the TS, namely the gastrocnemius, acts not only at the ankle joint, but also at the knee: if this muscle was more severely affected by pathological changes than the soleus, then clearly the disability experienced by the hemiparetic subjects would be more severe during gait, when the knee is extended and the gastrocnemius under greater tension than in this study, where the subjects were seated and the knee flexed. Given the similarity of the normal stiffness curves obtained in this study and those obtained in an earlier study ${ }^{10}$ where the measurements were performed with the knee almost fully extended, it is unlikely that the gastrocnemius could have such a dramatic effect on stiffness. Indeed, preliminary studies on the effects of knee angle indicate that significant changes in ankle stiffness occur only in the last $25^{\circ}$ of knee extension (unpublished observations). It would therefore seem justified to conclude that, although some change in the passive mechanical properties of the muscle fibres of the TS may have occurred, the major factor behind the increased resistance to dorsiflexion at the paretic ankle is an increase in the passive stiffness of the Achilles tendon.

This work was supported by the Deutsche Forschungsgemeinschaft. We thank Professors J Noth and P M H Rack for their helpful comments on this study.

1 Lance JW. Pathophysiology of spasticity and clinical experience with Baclofen. In: Feldman RG, Young RR Koella KP, eds. Spasticity: disordered motor control. Chicago: Year Book medical, 1980:185-203.

2 Dietz V, Quintern J, Berger W. Electrophysiological studies of gait in spasticity and rigidity: evidence that altered mechanical properties of muscle contribute to hypertonia. Brain 1981;104:431-9.

3 Dietz V, Ketelsen U-P, Berger W, Quintern J. Motor unit involvement in spastic paresis: relationship between leg muscle activation and histochemistry. J Neurol Sci 1986;75:89-103.

4 Hufschmidt A, Mauritz K-H. Chronic transformation of muscle in spasticity: a peripheral contribution to increased tone. J Neurol Neurosurg Psychiatry 1985;48:676-85.

5 Thilmann AF, Fellows SJ, Garms E. The mechanism of spastic muscle hypertonia: variation in reflex gain over time course of spasticity. Brain 1991 (In press).

6 Sim-Fook L, Hodgson AR. A comparison of foot forms among the non-shoe and shoe-wearing Chinese population. J Bone Joint Surg 1958;40A:1058-62.

7 Segesser B, Stüssi E, Stacoff A, Kälin X, Ackermann R. Torsin-ein neues Konzept im Sportschuhbau: Die Bewegungsexkursion des Fußes bei sportlichen Belastungen-Anatomische und biomechanische Betrachtungen
und ihre Auswirkungen auf den Sportschuhbau. Sport- 
verletzung Sportschaden 1989;3:167-82.

8 Regnauld B. The foot: pathology, aetiology, semiology, clinical investigation and therapy. Berlin: Springer Verlag, 1986:124.

9 Fellows SJ, Ross HF, Thilmann AF. Mechanical alterations in the flexibility of the spastic human ankle joint. J Physiol 1990;420:83P.

10 Weiss PL, Kearney RE, Hunter IW. Position dependence of ankle joint dynamics-I. Passive mechanics. J Biomech 1986;19:727-35.

11 Sutherland DH, Nagy JL. Measurement of gait movements from motion picture film. $J$ Bone Joint Surg 1972;54A:787-97.

12 Fellows SJ, Garms E, Thilmann AF. Transient and maintained phasic reflex components in spastic human maintained phasic reflex componen
subjects. J Physiol 1989;415:60P.

13 Hamilton JJ, Ziemer LK. Functional anatomy of the human ankle and foot. In: Kiene RH, Johnson KA, eds. American Academy of Orthopedic Surgeons symposium on the foot and Academy of Orthopedic Surgeons symposium

14 Rack PMH. Stretch reflexes in man: the significance of tendon compliance. In: Barnes WJP, Gladden MH, eds. Feedback and motor control in invertebrates and vertebrates. London: Croon Helm, 1985:217-29.

15 Bojsen-Moller F. The human foot-a two speed construc- tion. In: Asmussen E, Jorgensen K, eds. Biomechanics VIA Proc sixth International Congress of Biomechanics. A, Proc sixth International Congress of Biom

$16 \mathrm{Ker}$ RF. Dynamic tensile properties of the plantaris tendon of sheep (Ovis aries). J Exper Biol 1981;93:283-302.

17 Rack PMH, Ross HF, Thilmann AF, Walters DKW. Reflex responses at the human ankle: the importance of tendon compliance. J Physiol 1983;344:502-24.

18 Akeson WH, Woo SLY, Amiel D, Coutts RD, Daniel DD. The connective tissue response to immobility: Biomechanical changes in periarticular connective tissue of the immobilised Rabbit knee. Clinical Orthopaedics and Related Research 1973;93:356-62.

19 Neuberger A, Slack HGB. The metabolism of collagen from liver, bones, skin and tendon in the normal rat. Biochemical Journal 1953;53:47.

20 Elliot $\mathrm{DH}$. Structure and function of mammalian tendon. Biological Reviews 1965;40:392-421.

21 Lakie M, Walsh EG, Wright GW. Control and postural thixotropy of the forearm muscles: changes caused by cold. J Neurol Neurosurg Psychiatry 1986;49:69-76.

22 Lightfoot E, Verrier M, Ashby P. Neurophysiological effects of prolonged cooling of the calf in patients with complete spinal transection. Physical Therapy 1975;55:251-8. 